\title{
Social Function and Cognitive Status: Results from a US Nationally Representative Survey of Older Adults
}

\author{
Ashwin A. Kotwal, M.D., M.S. ${ }^{7}$, Juyeon Kim, Ph.D. ${ }^{2}$, Linda Waite, Ph.D. ${ }^{3}$, and William Dale, M.D. , Ph.D. ${ }^{4}$ \\ 'Department of Medicine, Brigham and Women's Hospital, Boston, MA, USA; ²Department of Urban Sociology, University of Seoul, Seoul, South \\ Korea; ${ }^{3}$ Department of Sociology, University of Chicago, Chicago, IL, USA; ${ }^{4}$ Department of Medicine, University of Chicago, Chicago, IL, USA.
}

BACKGROUND: An early sign of cognitive decline in older adults is often a disruption in social function, but our understanding of this association is limited.

OBJECTIVE: We aimed to determine whether those screening positive for early stages of cognitive impairment have differences across multiple dimensions of social function and whether associations differ by gender.

DESIGN: United States nationally representative cohort (2010), the National Social life, Health, and Aging Project (NSHAP).

PARTICIPANTS: Community-dwelling adults aged 6290 years $(N=3,310)$ with a response rate of $76.9 \%$.

MAIN MEASURES: Cognition was measured using a survey adaptation of the Montreal Cognitive Assessment categorized into three groups: normal, mild cognitive impairment (MCI), and dementia. We measured three domains of social relationships, each comprised of two scales: network structure (size and density), social resources (social support and social strain), and social engagement (community involvement and socializing). We used multiple linear regression to characterize the relationship of each social relationship measure to cognition.

KEY RESULTS: Individuals screened as at risk for MCI and early dementia had smaller network sizes by 0.3 and 0.6 individuals $(p<0.001)$, and a $10 \%$ and $25 \%$ increase in network density $(p<0.001)$, respectively. For social resources, individuals at risk for MCI and dementia had $4 \%$ and $14 \%$ less social strain $(p=0.01)$, but only women had $3 \%$ and $6 \%$ less perceived social support $(p=0.013)$, respectively. For social engagement, individuals screened positive for MCI and dementia had $8 \%$ and $19 \%$ less community involvement $(p=0.01)$, but only men had $8 \%$ and $13 \%$ increased social involvement with neighbors and family members $(p<0.001)$, respectively.

CONCLUSION: Changes in social functioning provide an early indication to screen for cognitive loss. Recognition that early cognitive loss is associated with differences in social function can guide counseling efforts and help identify social vulnerabilities to ease the transition to overt dementia for both patients and caregivers.

Received October 19, 2015

Revised March 9, 2016

Accepted March 28, 2016

Published online April 29, 2016
KEY WORDS: aging; cognition; social relationships; community dwelling; MoCA.

$\mathrm{J}$ Gen Intern Med 31(8):854-62

DOI: $10.1007 / \mathrm{s} 11606-016-3696-0$

(c) Society of General Internal Medicine 2016

\section{INTRODUCTION}

An early sign of cognitive decline in older adults can be disruption of social activities, including loss of community connections and altered interactions with family and friends. Changes in social function in older adults can reduce quality of life, ${ }^{1,2}$ impede independent living, ${ }^{3}$ and increase mortality. ${ }^{4,5}$ An improved understanding of the relationship of cognitive decline with social function could help identify areas where older adults need more support to address social vulnerabilities and preserve independence. It could also help target cognitive screening to those with early signs of impairment.

Most research on cognition and social relationships examines whether improved social relationships can delay dementia onset. ${ }^{6}$ Longitudinal studies show that larger social networks, ${ }^{7,8}$ access to social resources, ${ }^{9,10}$ and community engagement reduce dementia diagnoses. ${ }^{11-17}$ Hypotheses on mechanisms by which social relationships can reduce cognitive decline include: delay of cognitive atrophy ("use it or lose it"), ${ }^{18}$ higher cognitive reserve, ${ }^{19}$ and preventing stress-related cognitive declines. ${ }^{6}$ This is likely a bidirectional relationship, where cognitive decline can lead to altered social relationships. $^{20-22}$

However, there is less information on the relationship of social function with earlier stages of cognitive decline such as mild cognitive impairment (MCI) in community-based samples. Cognitive assessments have relied on dementia diagnoses or have used screening tools, such as the Short Portable Mental Status Questionnaire (SPMSQ), ${ }^{21,23,24}$ or the MiniMental Status Exam (MMSE), ${ }^{8,10,25}$ which are relatively insensitive to early impairment. ${ }^{26-28}$ Clinicians would benefit from understanding whether early stages of impairment are associated with altered social relationships.

Previous studies focus on limited, isolated dimensions of social function rather than using a broad set of interrelated factors. To understand the full clinical consequences of lower cognition for social relationships, multiple aspects of social function should be assessed. ${ }^{4,14}$ For example, in examining 
social network structure, network sizes are smaller for those with cognitive impairment, ${ }^{7,8}$ suggesting less social support and higher potential caregiver burden. However, it is unclear whether composition of the network relationships also change. ${ }^{29}$ Smaller, denser networks with more communication and coordination may help provide support. Regarding social resources, individuals with lower cognition may be less able to mobilize social resources, but it is unclear where these perceived losses of support occur and if individuals continue to perceive demands from friends and family. Inability to utilize social resources may indicate a barrier to independent living. For social engagement, one might expect decreased community involvement at lower cognitive status, ${ }^{10,12,13}$ but less is known regarding social isolation at home. Less interaction with friends, family and the community is often the most noticeable social sign of cognitive impairment, and a better characterization of such differences could provide clues on when to screen for cognitive loss.

Evidence suggests that social relationships differ substantially by gender, ${ }^{30-33}$ and therefore changes in cognition likely impact social relationships in a different way by gender. However, few studies have examined this; stronger associations between cognition and social engagement exist for women, ${ }^{12,13,21,34}$ but nothing is known regarding other social domains. Knowing about gender differences linking cognitive function and other domains of social relationships could help clinicians better manage older patients with early cognitive impairment.

Using a nationally representative sample of communitydwelling older adults, the National Social life Health and Aging Project (NSHAP; Wave 2), we examine the link between cognition and multiple social relationships. Our goal is to provide those caring for older adults with a richer description of social differences at earlier stages of cognitive impairment, which may differ by gender. We hypothesize that lower cognition, even at early stages, is associated with smaller and denser networks, less access to social resources, and less engagement with family, friends, and community. We further hypothesize the association of cognition with social relationships to be weaker for women because of their greater experience in mobilizing social resources.

\section{METHODS}

Study Sample. We used the NSHAP Wave 2 (W2) sample, an in-home, nationally representative probability sample of older adults from households across the United States, collected between August 2010 and May 2011. ${ }^{35}$ As a sampling strategy, NSHAP-W2 included co-resident spouses and partners, yielding 3,377 respondents aged 62-90 years old. The weighted overall response rate was $76.9 \%$. Individuals unable to complete the interview, due to physical or cognitive limitations, were not enrolled. After the in-person survey was completed, respondents were also given a paper-and-pencil questionnaire with additional questions on social life to be mailed into the collection center, which was completed by $88.6 \%$ of participants. Individuals with missing values for key covariates were excluded $(n=67)$, yielding a final sample of 3,310 individuals. All respondents provided written informed consent and the protocol was approved by the institutional review boards at the University of Chicago and National Opinion Research Center (NORC).

Social Relationships. We evaluated three dimensions of social relationships: social network structure, social resources, and social engagement. First, we examined two structural aspects of social networks: size and density. Size was determined using NSHAP's network roster, asking respondents to name up to five persons with whom he or she "discussed important matters" within the past 12 months. ${ }^{35}$ This method elicits the number of influential individuals in respondents' lives. ${ }^{36}$ Density describes the overall degree of involvement of all members of the network and how close-knit a respondent's social context is. It was calculated as a respondent's frequency of interaction with each network member plus the frequency of interactions among each possible pair of network members, divided by network size. Density values are continuous between a range of 0 and 1 , where a value of 1 indicates that all possible pairs of network members have the maximum contact, and a value of 0 indicates no contact between any of the possible pairs of network members. ${ }^{36}$

Second, we examined social resources and demands that accompany social relationships: perceived social support and perceived social strain. Perceived social support was measured by asking whether the respondent could (independently): 1) rely on and 2) open up to their spouse, family, or friends. Each of these six items had possible responses of "Never" ( 0 points), "Rarely" (1 point), "Sometimes" (2 points), or "Often" (3 points). Sources were combined to yield a composite scale ranging from 0 to 18 points (Cronbach's $\alpha=0.64$ ), with 0 indicating no social support and 18 representing frequent social support from all three sources. ${ }^{37}$ Perceived social strain was evaluated by asking how often the respondent's spouse, family, or friends: 1) make too many demands, 2) criticize you, or 3) get on your nerves. These nine items combined into a composite scale ranging from 0 to 27 points (Cronbach's $\alpha=0.73$ ), with 0 representing no social strain and 27 points representing frequent social strain from all three sources. Unmarried adults received scale scores for friends and family only, and therefore, friends and family item scores were averaged and rescaled to the full scale for comparability with the married.

Third, we examined two aspects of social engagement: community involvement $(n=2,853)$ and socializing with relatives and friends $(n=2,880)$. Items for each scale were included in the leave-behind questionnaire, yielding smaller sample sizes. Community involvement was measured using three items asking about the 
frequency in the past 12 months of: 1) attending religious services, 2) attending community group meetings, and 3) volunteering. The three items were combined into a scale ranging from 0 to 18 points (Cronbach's $\alpha=0.71$ ), where a value of 0 indicates no participation and a value of 18 indicates frequent participation in all three activities. Socializing with relatives and friends was measured using two items describing how frequently in the last year respondents interacted socially with: 1) friends or relatives; and 2) neighbors. These items were combined to create a $0-6$ point scale, with 0 indicating low socialization and 6 indicating high socialization.

Independent Variable-Cognitive Status. The Montreal Cognitive Assessment (MoCA) is a screening tool designed to differentiate cognitive changes of normal aging from MCI and early dementia in clinical populations. ${ }^{38}$ Following extensive testing, an 18 -item survey adaptation of the Montreal Cognitive Assessment (MoCA-SA) was included in NSHAP-W2. ${ }^{28}$ The MoCA-SA was designed for administration by nonmedical personnel and to reduce respondent burden within the context of a large, time-limited national survey, while preserving the MoCA's sensitivity to a range of cognitive abilities. MoCA-SA scores are highly correlated with the MoCA and scores can be accurately converted into MoCA scores. ${ }^{39}$ The specific items included are: 1) Orientation: date and month (2 points total); 2) Executive function: abstraction-similarity of watch and ruler (1 point), modified Trails-b (1 point); 3 ) Visuospatial skills: clock-contour, numbers, and hands (3 points total); 4) Memory: 5-word delayed recall (5 points); 5) Attention: forward digits (1 point), backward digits (1 point), subtract $7 \mathrm{~s}$ (3 points); and 6) Language: naming rhinoceros (1 point), phonemic fluency-words with the letter "F" (1 point for $>10$ words in $60 \mathrm{~s}$ ), and sentence repetition (1 point).

Questions remain regarding most appropriate cutoffs from the MoCA to screen for MCI or dementia; studies from selected clinical samples favor higher cutoff values to define MCI, ${ }^{38,40,41}$ compared to community-based studies, ${ }^{42-44}$ which accept lower cutoffs. We divided the scale into three categories based on community samples: $>22$ points (normal), 18-22 points (screen positive for MCI), $<18$ points (screen positive for dementia). ${ }^{43}$ These latter categories represent groups that would screen positive on the MoCA for likely cognitive impairment recommending neuropsychiatric testing. Sensitivity analyses of MoCA-SA both as a continuous variable and as a categorical variable with higher cutoffs yielded similar results (available by request).

Covariates. Covariates were chosen based on prior work identifying them as potential confounders. ${ }^{12,17,45} \mathrm{We}$ included self-reported age, gender (male or female), ethnicity (White, African American, Hispanic, or other), education (less than high school, high school or its equivalent, some college or vocational degree, and college degree or higher), and marital status (married, divorced or separated, widowed, and never married). Health status was measured using self-rated physical health, grouped into dichotomous categories of "Excellent/ Very Good/Good" or "Fair/Poor," and the NSHAP Comorbidity index, as detailed elsewhere. ${ }^{46}$ Depressive symptoms were measured using an adaption of the Center for Epidemiological Studies-Depression (CES-D) scale. ${ }^{47}$ Health behaviors were included as controls in the analysis, including tobacco use, exercise, and alcohol consumption. ${ }^{48}$

Statistical Analysis. The association of each social relationship variable with cognitive status was assessed in six separate multiple linear regression models. Each included covariates (age, gender, education, ethnicity, marriage, selfrated health, comorbidities, depressive symptoms, smoking, exercise, and alcohol use). We tested for interactions between cognitive status and gender in each model and included

Table 1. Weighted Sample Demographic and Health Characteristics $(n=3310)$

\begin{tabular}{|c|c|c|}
\hline & Variables & Percent (\%) \\
\hline \multicolumn{3}{|l|}{ Cognition } \\
\hline \multirow[t]{3}{*}{ Cognitive Status } & Normal (MoCA > 22) & 58.9 \\
\hline & MCI (MoCA 18-22) & 25.8 \\
\hline & Dementia (MoCA < 18) & 15.3 \\
\hline \multicolumn{3}{|l|}{ Demographic/SES } \\
\hline Gender & Female & 51.8 \\
\hline \multirow[t]{4}{*}{ Education (\%) } & $<\mathrm{HS}$ & 16.5 \\
\hline & HS/GED & 25.9 \\
\hline & Some College & 32.3 \\
\hline & Bachelor+ & 25.3 \\
\hline \multirow[t]{4}{*}{ Ethnicity/Race } & White & 80.7 \\
\hline & AA & 9.8 \\
\hline & Hispanic & 6.8 \\
\hline & Other & 2.7 \\
\hline \multirow[t]{4}{*}{ Marital Status } & Married & 64.7 \\
\hline & Divorced & 10.6 \\
\hline & Widowed & 22.2 \\
\hline & Single & 2.6 \\
\hline \multicolumn{3}{|l|}{ Health behaviors } \\
\hline \multirow[t]{3}{*}{ Smoking } & Never & 40.8 \\
\hline & Former & 45.9 \\
\hline & Current & 13.3 \\
\hline Alcohol $^{\dagger}$ & High & 11.1 \\
\hline Physical & Never & 24.4 \\
\hline \multirow{5}{*}{ Activity $^{\ddagger}$} & $<1$ month & 8.7 \\
\hline & $1-2 /$ month & 8.3 \\
\hline & $1-2 /$ week & 16.7 \\
\hline & 3-4/week & 20.2 \\
\hline & 5+/week & 21.6 \\
\hline \multicolumn{3}{|l|}{ Health Characteristics } \\
\hline Self-Rated Health & Poor/Fair & 25.3 \\
\hline Depressive Symptoms ${ }^{\S}$ & High $(9+)$ & 20.8 \\
\hline
\end{tabular}

Abbreviations: G Good, VG Very Good, Exc Excellent, MoCA Montreal Cognitive Assessment

${ }^{\dagger}$ Two criteria used to define problem drinking include: 1) > 14 drinks/ week for men or $>9$ drinks/week for women, or 2) $\geq 3$ binge drinking episodes (4 or more drinks in one day) in last 3 months

${ }^{F}$ Physical Activity defined as $>30$ min of vigorous physical activity (sports, exercise classes, heavy housework, physical labor) in last 12 months

${ }^{\xi}$ Depressive symptoms measured by 11 separate items with range of 0 to 33 
statistically significant $(p<0.05)$ interaction terms in the final individual models. Model assumptions about linearity, variable independence, multicollinearity, and normality of residuals were assessed and met. ${ }^{49}$ Beta coefficients are reported, as are $95 \%$ confidence intervals. Adjusted marginal probability estimates derived from the final models are used to estimate the level of social functioning for those screening positive for dementia, MCI, and normal cognition. All statistical analyses utilized sampling weights to ensure representativeness and were performed using Stata 12.1. ${ }^{50,51}$

\section{RESULTS}

Sample characteristics are shown in Table 1. Average age was 72.4 years $(\mathrm{SD}=7.3)$, approximately $52 \%$ were women, and $65 \%$ were married. The mean number of comorbidities was $2.7(\mathrm{SD}=2.0)$. The mean estimated MoCA score was 22.6 $(\mathrm{SD}=4.4)$; using community cutoffs, $26 \%$ screened as at risk for MCI (18-22) and $15 \%$ screened as at risk for dementia (< 18). Bivariate associations between cognition and social relationships are shown in Table 2.

Multiple regression results are shown in Table 3. After adjustment for covariates, individuals who screened as at risk for MCI and dementia had smaller network sizes by 0.3 individuals and 0.6 individuals, respectively, as compared with individuals screened as normal $(p<0.001)$ (Fig. 1a). Lower cognition was associated with a $10 \%$ and $25 \%$ increase in density for those screened for MCI and dementia, respectively $(p<0.001)$ (Fig. 1b). Subsequent analysis showed a higher proportion of family members in their network than those screened as normal (65\% in normal vs. $73 \%$ in dementia).

The association of cognitive status with social support differed by gender ( $p=0.013$ ) (Fig. 2a). Overall, women with lower cognitive scores perceived less social support $(p=0.01)$, but there was no difference for men $(p=0.99)$. Women perceived the largest decrease in support from friends compared with family and their spouse $(p=0.04)$, whereas men perceived increased support from their spouse $(p=0.01)$. Both men and women with lower cognitive scores perceived less social strain than those screened as normal $(p=0.01)$, mainly from their spouse (Fig. 2b).

For social engagement, individuals with lower cognitive scores reported lower community involvement than those screened as normal ( $p=0.01)$ (Fig. 3a); there was decreased participation in group activities $(p<0.001)$ and volunteer work $(p=0.05)$, whereas religious involvement remained similar. In contrast, community involvement increased with age (Fig. 3b). Finally, the association of cognition with socializing differed by gender $(p<0.001)$ (Fig. 3c); men with lower cognitive scores socialized more with friends and relatives $(p=0.001)$, but there was no association for women $(p=0.80)$.

\section{DISCUSSION}

In a nationally representative sample of community-dwelling older adults, the NSHAP-W2, we found that lower cognition

Table 2. Social Relationships in Full Sample and by Cognitive Status

\begin{tabular}{|c|c|c|c|c|}
\hline & \multirow{3}{*}{$\begin{array}{l}\text { Overall } \\
\text { Mean }(95 \% \mathrm{CI})\end{array}$} & \multicolumn{3}{|l|}{ Cognition $^{*}$} \\
\hline & & \multirow{2}{*}{$\frac{\text { Normal }}{\text { Mean (95 \% CI) }}$} & \multirow{2}{*}{$\frac{\text { MCI }}{\text { Mean }(95 \% \text { CI })}$} & \multirow{2}{*}{$\frac{\text { Dementia }}{\text { Mean (95 \% CI) }}$} \\
\hline & & & & \\
\hline \multicolumn{5}{|l|}{ Network Structure } \\
\hline Network Size ${ }^{\dagger}(n=3310)$ & $3.7(3.6,3.8)$ & $4.0(3.9,4.1)$ & $3.6(3.4,3.7)$ & $3.1(2.8,3.3)$ \\
\hline Network Density $(n=3277)$ & $0.33(0.31,0.34)$ & $0.29(0.28,0.31)$ & $0.35(0.32,0.38)$ & $0.43(0.39,0.46)$ \\
\hline \multicolumn{5}{|l|}{ Social Resources } \\
\hline Social Support ${ }^{\S}(n=3307)$ & $14.1(13.9,14.3)$ & $14.6(14.4,14.7)$ & $13.9(13.6,14.1)$ & $12.5(11.8,13.2)$ \\
\hline Social Strain" $(n=3283)$ & $8.3(8.1,8.5)$ & $8.7(8.4,8.9)$ & $8.2(8.0,8.5)$ & $7.0(6.5,7.5)$ \\
\hline \multicolumn{5}{|l|}{ Social Engagement } \\
\hline Community $(n=2853)$ & $8.1(7.7,8.5)$ & $8.7(8.3,9.1)$ & $7.4(6.7,8.2)$ & $6.3(5.7,7.0)$ \\
\hline Socializing $^{\#}(n=2880)$ & $642(4.1,6.3)$ & $4.3(4.2,4.3)$ & $4.2(4.0,4.4)$ & $4.0(3.8,4.2)$ \\
\hline
\end{tabular}

Abbreviations: CI confidence interval, MCI mild cognitive impairment

* Cognitive status is determined using MoCA cutoffs of normal (> 22), MCI (18-22), and dementia (< 18)

${ }^{\dagger}$ Network Size has a range of 0 to 5 and refers to persons with whom the respondent "discussed important matters" within the past 12 months

${ }^{*}$ Network density values are continuous between a range of 0 and 1 , where a value of 1 indicates that all possible pairs of network members have some contact. Density is a function of the respondents' frequency of interaction with each network member and their perception of the frequency of interactions among each possible pair of network members, divided by network size

${ }^{s}$ Perceived social support has a range of 0 to 18 and is measured from six items asking whether the respondent could: 1) rely on, or 2) open up to their spouse, family, or friends

"Social strain has a range of 0 to27 and is measured from nine items asking how often the respondent's spouse, family, or friends: 1) make too many demands, 2) criticize you, or 3) get on your nerves

${ }^{\top}$ Community involvement has a range of 0 to 18, and includes three items assessing the frequency in the past 12 months of: 1) attending religious services, 2) attending organized group meetings, and 3) volunteering

${ }^{\#}$ Socializing has a range of 0 to 6 and includes two items assessing the frequency with which the respondent got together socially in the last year with:1) friends or relatives, and 2) neighbors 
Table 3. Multiple Regression Models of Cognitive Status by Social Relationship Measures

\begin{tabular}{|c|c|c|c|c|c|c|}
\hline & \multirow[b]{2}{*}{ MCI } & \multirow[b]{2}{*}{ Dementia } & \multirow{3}{*}{$p$ value } & \multicolumn{3}{|c|}{ Interaction with Female Gender } \\
\hline & & & & MCI & Dementia & $p$ value \\
\hline & Coefficient (CI) & Coefficient (CI) & & Coefficient (CI) & Coefficient (CI) & \\
\hline \multicolumn{7}{|l|}{ Network Structure } \\
\hline Network Size $(n=3310)$ & $-0.23(-0.43,0.03)$ & $-0.56(-0.81,-0.30)$ & $<0.001$ & - & - & \\
\hline Network Density $(n=3277)$ & $0.03(0.004,0.12)$ & $0.08(0.05,0.12)$ & 0.002 & - & - & \\
\hline \multicolumn{7}{|l|}{ Social Resources } \\
\hline Social Support $(n=3307)$ & $0.02(-0.33,0.37)$ & $0.00(-0.45,0.46)$ & 0.99 & $-0.48(-0.94,0.02)$ & $-0.74(-1.29,-0.19)$ & 0.013 \\
\hline Spouse & $0.01(-0.11,0.13)$ & $0.24(0.08,0.40)$ & 0.01 & $0.04(-0.13,0.21)$ & $-0.26(-0.48,-0.04)$ & 0.04 \\
\hline Family & $-0.04(-0.2,0.12)$ & $-0.05(-0.26,0.16)$ & 0.85 & $-0.16(-0.38,0.05)$ & $-0.21(-0.47,0.04)$ & 0.14 \\
\hline Friends & $0.03(-0.17,0.23)$ & $-0.09(-0.35,0.17)$ & 0.67 & $-0.26(-0.52,-0.004)$ & $-0.33(-0.64,-0.02)$ & 0.04 \\
\hline Social Strain $(n=3283)$ & $-0.30(-0.71,0.10)$ & $-1.15(-1.88,-0.42)$ & 0.011 & & - & \\
\hline Spouse & $-0.08(-0.27,0.12)$ & $-0.47(-0.78,-0.16)$ & 0.02 & & & \\
\hline Family & $-0.08(-0.21,0.04)$ & $-0.14(-0.36,0.08)$ & 0.31 & & & \\
\hline Friends & $-0.02(-0.12,0.08)$ & $-0.08(-0.23,0.06)$ & 0.52 & & & \\
\hline \multicolumn{7}{|l|}{ Social Engagement } \\
\hline Community $(n=2853)$ & $-0.67(-1.28,-0.06)$ & $-1.52(-2.54,-0.49)$ & 0.011 & - & & \\
\hline Groups & $-0.19(-0.32,-0.06)$ & $-0.45(-0.64,-0.26)$ & $<0.001$ & & & \\
\hline Volunteer & $-0.15(-0.30,-0.01)$ & $-0.24(-0.45,-0.03)$ & 0.05 & & & \\
\hline Religious & $-0.04(-0.18,0.10)$ & $-0.11(-0.36,0.13)$ & 0.64 & & & \\
\hline Socializing $(n=2880)$ & $0.30(0.01,0.59)$ & $0.53(0.25,0.81)$ & 0.001 & $-0.36(-0.7,-0.1)$ & $-0.66(-1.0,-0.3)$ & $<0.001$ \\
\hline Family/Friends & $0.05(-0.06,0.18)$ & $0.17(0.03,0.31)$ & 0.043 & $-0.09(-0.23,0.05)$ & $-0.32(-0.49,-0.15)$ & 0.001 \\
\hline Neighbors & $0.24(0.03,0.46)$ & $0.35(0.13,0.56)$ & 0.004 & $-0.28(-0.52,-0.03)$ & $-0.32(-0.58,-0.06)$ & 0.017 \\
\hline
\end{tabular}

The reference group for cognition coefficients are those screened as "Normal" Abbreviations: $C I 95 \%$ confidence interval, MCI mild cognitive impairment,

Each linear regression model adjusts for age, gender, education, ethnic group, marital status, self-rated health, comorbidities, depressive symptoms, smoking, exercise, and alcohol

was associated with significant differences across multiple dimensions of social functioning. Associations were apparent even in those screening positive for early stages of cognitive impairment, and associations often differed by gender.

As hypothesized and consistent with prior literature, individuals with lower cognitive scores had smaller social networks. ${ }^{7,14}$ In addition, those with lower cognitive scores had networks that were denser, more interconnected, and contained a higher proportion of relatives. One possible explanation for this finding is that lower cognition hinders the maintenance of diverse and distant network ties, leading to smaller and denser networks. ${ }^{31}$ Alternatively, this difference may reflect compensatory changes in network structure in response to cognitive difficulties-more
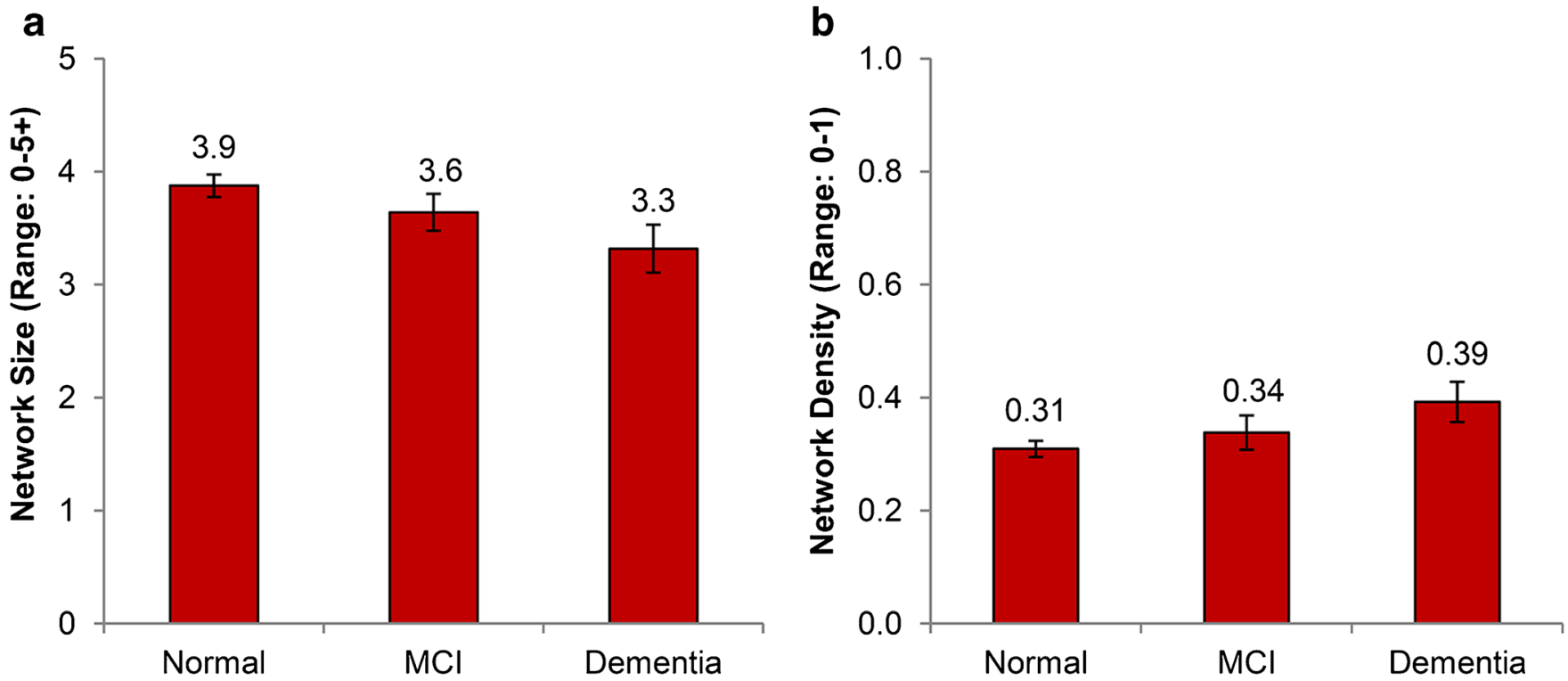

Figure 1. Adjusted marginal estimates of the association between cognitive status and social relationship structure, including a) network size and b) network density. Marginal estimates are from separate linear regression models adjusted for age, gender, education, ethnic group, marital status, self-rated health, comorbidities, depressive symptoms, smoking, exercise, and alcohol. Bars represent $95 \%$ confidence intervals. Abbreviations: MCI - mild cognitive impairment. 
a

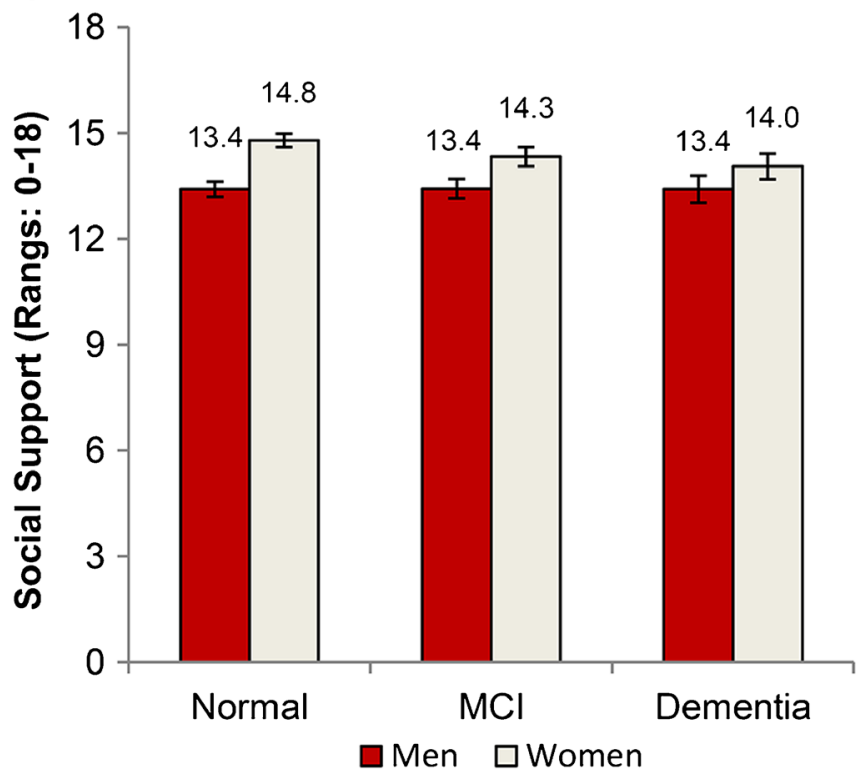

b

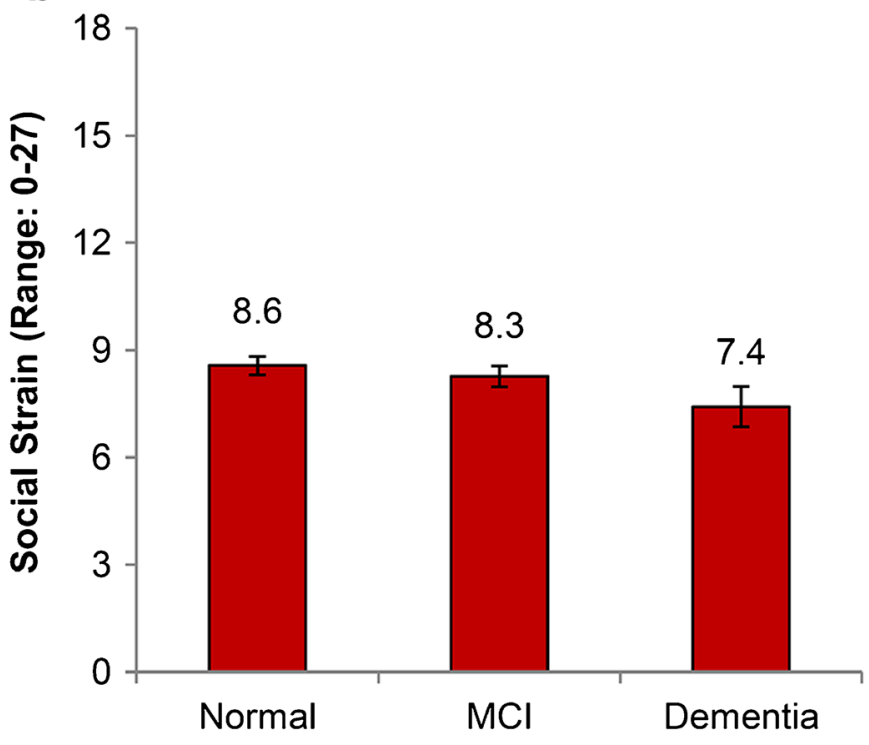

Figure 2. Adjusted marginal estimates of the association between cognitive status and social resources, including a) social support, b) social strain. Marginal estimates are from separate linear regression models adjusted for age, gender, education, ethnic group, marital status, selfrated health, comorbidities, depressive symptoms, smoking, exercise, and alcohol. Bars represent $95 \%$ confidence intervals. Abbreviations: MCI - mild cognitive impairment.

densely-linked, family-centered networks may help in monitoring and supporting the health of an impaired individual. ${ }^{52}$ A longitudinal assessment of network density is needed to further test these mechanisms.

Results supported our hypothesis that lower cognition is associated with decreased access to social resources, with notable gender differences. Women with lower cognition scores perceived significantly less social support than women with higher scores, particularly from friends. Conversely, men with lower cognition scores perceived increased spousal support. Prior literature suggests women are better able to mobilize social support when needed, particularly in a health context. ${ }^{30} \mathrm{We}$ speculate that lower cognitive status in women, even at its mildest, may decrease their ability to mobilize and maintain social support. Expanding on prior literature, both men and women reported less perceived social strain with lower cognitive status, consistent with network members placing fewer social demands on them. ${ }^{14}$

Consistent with our hypothesis based on prior literature, lower cognition was associated with lower levels of community involvement. Importantly, this is distinct from most older adults who have increased levels of community engagement with age. ${ }^{53}$ This was true even for individuals screening positive for MCI, suggesting an early indicator of cognitive loss. We also hypothesized that lower cognition would be associated with social isolation from family and friends, which was not the case. Instead, men with lower cognitive status socialized more with friends and neighbors, while women showed no differences. Perhaps as men experience cognitive decline, their overall network size contracts, but they rely more on proximal relations, such as family, neighbors, and close friends. ${ }^{30}$ Alternatively, close friends and relatives may "check in on" men with cognitive difficulties more frequently. We note that results held even after controlling for marital status, suggesting this to be unrelated to women in the sample outliving men.

Our results have clinical implications. First, disruptions in social function may be an indication for providers to conduct cognitive screens like the MoCA, even when other known signs of cognitive impairment are not apparent. Specific social clues supported by our data include having fewer confidantes, increasing reliance on family, and women perceiving less support from friends. Less participation in community activities may also be a reason to screen for cognitive impairment, since age is typically associated with increased participation. Second, the loss of diverse network ties and a reliance on denser, more interconnected networks may increase caregiver burden, which is associated with poor outcomes for both the patient and caregiver. $^{54-56}$ Consequently, identifying caregiver stress and mobilizing support at earlier stages of cognitive loss may help ease the transition to overt dementia, should it occur. Finally, clinicians can counsel patients and families on social function changes that may occur at early stages of cognitive loss. For example, women with early cognitive loss experience greater perceived losses in social support, and providers should connect these women with social resources and support. ${ }^{56}$ 

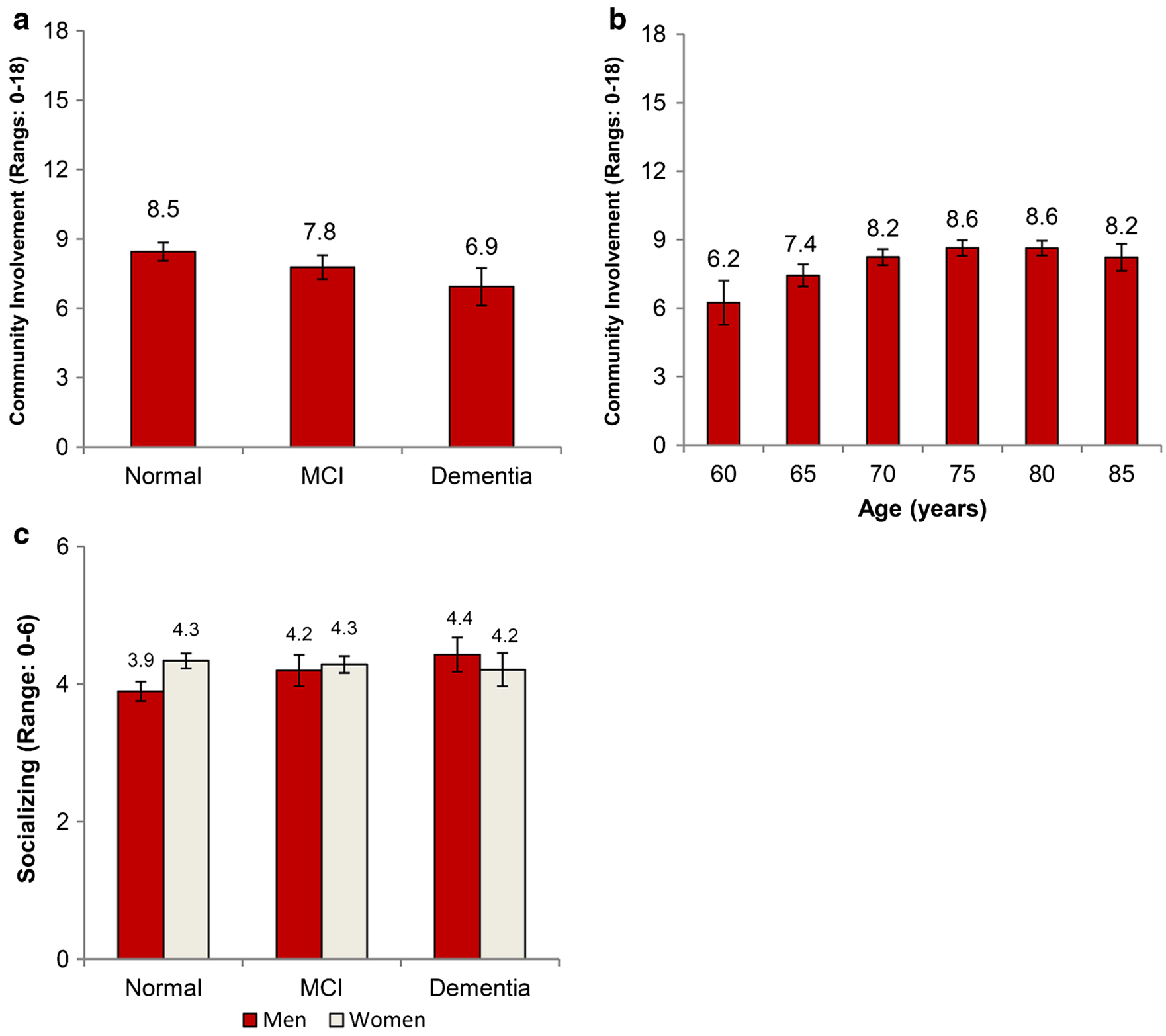

Figure 3. Adjusted marginal estimates of the association between cognitive status and social relationships. Panel a shows the association of community involvement with cognition; this contrasts with Panel b, which shows the association of community involvement with age. Panel $c$ shows the association between socializing and cognition with the interaction with gender. Marginal estimates are from separate linear regression models adjusted for age, gender, education, ethnic group, marital status, self-rated health, comorbidities, depressive symptoms, smoking, exercise, and alcohol. Bars represent $95 \%$ confidence intervals. Abbreviations: MCI - mild cognitive impairment.

Our study has limitations. First, we do not have full neuropsychological testing for our large sample, and thus cannot provide formal diagnoses of MCI or dementia. However, we used a well-tested survey adaptation of a screening tool, the MoCA-SA, ${ }^{28,39}$ as well as appropriate cutoffs for a community sample. ${ }^{42-44}$ A sensitivity analysis utilizing both a continuous cognitive measure and multiple different cutoffs yielded similar associations with social variables (data available on request). Second, the study was cross-sectional, reducing our ability to make causal claims, particularly about whether social or cognitive changes occurred first. This will have to be addressed with future waves of NSHAP utilizing longitudinal data or other data. As has been noted elsewhere, both longitudinal and cross-sectional data have complementary strengths and weaknesses for assessing causal relationships in cognition, in large part due to the long prodromal period of cognitive impairment and a lack of information on the timing of critical early neurologic events. ${ }^{57}$ Third, the data is focused on community-dwelling, high-functioning older adults, and therefore does not assess the association of social relationships with more profound cognitive impairment. Since cognitive status likely factors into the ability to participate in a 2$\mathrm{h}$ survey, the exclusion of those unable to do so further limits generalizability.

In conclusion, in a large, nationally representative sample, we found that lower cognitive scores are associated with important 
differences in social function. These associations sometimes vary by gender. Results inform the counseling of patients and families at risk for caregiver stress, targeted screening of older adults at risk for early cognitive loss, and identifying social vulnerabilities amenable to intervention and resources.

Acknowledgments: This study was supported by the Clinical and Translational Science Award (CTSA) TL1 pre-doctoral training grant. This work was supported by funding for MERIT Award R37 AG030481 (PI: Waite) from the National Institute on Aging, and from the National Institutes of Health, including the National Institute on Aging, the Office of Women's Health Research, the Office of AIDS Research, the Office of Behavioral and Social Sciences Research, and the National Institute on Child Health and Human Development for the National Health, Social Life, and Aging Project (NSHAP R01AG021487, R37AG030481), the NSHAP Wave 2 Partner Project (R01AG033903), and by NORC, which was responsible for the data collection. We greatly appreciate the very helpful comments of four anonymous reviewers on the manuscript and data analysis.

This work was presented as an oral presentation at the Neurological and Behavioral Sciences Session, American Geriatrics Society 2013 Annual Meeting, Dallas, TX.

Corresponding Author: Ashwin A. Kotwal, M.D., M.S.; Department of Medicine Brigham and Women's Hospital, Boston, MA 02115, USA (e-mail: akotwal@partners.org).

\section{Compliance with Ethical Standards:}

Conflict of Interest: The authors declare that they do not have a conflict of interest.

\section{REFERENCES}

1. Secker J, Hill R, Villeneau L, Parkman S. Concept forum promoting independence: but promoting what and how. Ageing Soc. 2003;23(3):375391.

2. Hillcoat-Nallétamby $\mathbf{S}$. The meaning of "independence" for older people in different residential settings. J Gerontol Ser B Psychol Sci Soc Sci. 2014;69(3):419.

3. Mack R, Salmoni A, Viverais-Dressler G, Porter E, Garg R. Perceived risks to independent living: the views of older, community-dwelling adults. The Gerontologist. 1997;37(6):729-736.

4. Berkman LF, Glass T, Brissette I, Seeman TE. From social integration to health: Durkheim in the new millennium. Soc Sci Med. 2000;51(6):843-857.

5. Sampson EL, Bulpitt CJ, Fletcher AE. Survival of community-dwelling older people: the effect of cognitive impairment and social engagement. J Am Geriatr Soc. 2009;57(6):985-991.

6. Fratiglioni L, Paillard-Borg $\mathbf{S}$, Winblad B. An active and socially integrated lifestyle in late life might protect against dementia. Lancet Neurol. 2004;3(6):343-353.

7. Fratiglioni L, Wang HX, Ericsson $\mathbf{K}$, Maytan M, Winblad B. Influence of social network on occurrence of dementia: a community-based longitudinal study. Lancet. 2000;355(9212): 1315.

8. Crooks VC, Lubben J, Petitti DB, Little D, Chiu V. Social network, cognitive function, and dementia incidence among elderly women. Am J Public Health. 2008;98(7):1221.

9. Andrew MK, Rockwood K. Social vulnerability predicts cognitive decline in a prospective cohort of older Canadians. Alzheimer's Dementia. 2010;6(4):319.

10. Barnes LL, De Leon CFM, Wilson RS, Bienias JL, Evans DA. Social resources and cognitive decline in a population of older African Americans and whites. Neurology. 2004;63(12):2322-2326.

11. Wang HX, Karp A, Winblad B, Fratiglioni L. Late-life engagement in social and leisure activities is associated with a decreased risk of dementia: a longitudinal study from the Kungsholmen project. Am J Epidemiol. 2002;155(12):1081-1087.

12. Zunzunegui Mv, Alvarado BE, Del Ser T, Otero A. Social networks, social integration, and social engagement determine cognitive decline in community-dwelling Spanish older adults. J Gerontol Ser B Psychol Sci Soc Sci. 2003;58(2):S93-S100.
13. Béland F, Zunzunegui M-V, Alvarado B, Otero A, del Ser T. Trajectories of cognitive decline and social relations. J Gerontol Ser B Psychol Sci Soc Sci. 2005;60(6):320-P330.

14. Seeman TE, Lusignolo TM, Albert M, Berkman L. Social relationships, social support, and patterns of cognitive aging in healthy, high-functioning older adults: MacArthur studies of successful aging. Health Psychol; Health Psychol. 2001;20(4):243.

15. Ertel KA, Glymour MM, Berkman LF. Effects of social integration on preserving memory function in a nationally representative US elderly population. Am J Public Health. 2008;98(7):1215-1220.

16. Saczynski JS, Pfeifer LA, Masaki K, et al. The effect of social engagement on incident dementia the Honolulu-Asia aging study. Am J Epidemiol. 2006; 163(5):433-440.

17. Seeman TE, Miller-Martinez DM, Merkin SS, Lachman ME, Tun PA, Karlamangla AS. Histories of social engagement and adult cognition: midlife in the US study. J Gerontol Ser B Psychol Sci Soc Sci. 2011;66(S1):i141-i152.

18. Hultsch DF, Hertzog C, Small BJ, Dixon RA. Use it or lose it: engaged lifestyle as a buffer of cognitive decline in aging? Psychol Aging. 1999; 14(2):245.

19. Bennett DA, Schneider JA, Tang Y, Arnold SE, Wilson RS. The effect of social networks on the relation between Alzheimer's disease pathology and level of cognitive function in old people: a longitudinal cohort study. Lancet Neurol. 2006;5(5):406-412.

20. Bosma H, van Boxtel MP, Ponds R, et al. Engaged lifestyle and cognitive function in middle and old-aged, non-demented persons: a reciprocal association? Z Gerontol Geriatr. 2002;35(6):575-581.

21. Thomas PA. Gender, social engagement, and limitations in late life. Soc Sci Med. 2011;73(9):1428-1435.

22. Green AF, Rebok G, Lyketsos CG. Influence of social network characteristics on cognition and functional status with aging. Int $\mathrm{J}$ Geriatric Psychiatry. 2008;23(9):972-978.

23. Bassuk SS, Glass TA, Berkman LF. Social disengagement and incident cognitive decline in community-dwelling elderly persons. Ann Intern Med. 1999; 131(3): 165.

24. Glei DA, Landau DA, Goldman N, Chuang Y-L, Rodriguez G, Weinstein M. Participating in social activities helps preserve cognitive function: an analysis of a longitudinal, population-based study of the elderly. Int $\mathrm{J}$ Epidemiol. 2005;34(4):864-871.

25. Holtzman RE, Rebok GW, Saczynski JS, Kouzis AC, Doyle KW, Eaton Ww. Social network characteristics and cognition in middle-aged and older adults. J Gerontol Ser B Psychol Sci Soc Sci. 2004;59(6):278284.

26. Friedman TW, Yelland GW, Robinson SR. Subtle cognitive impairment in elders with mini-mental state examination scores within the "normal'range. Int J Geriatric Psychiatry. 2011;27(5):463-471.

27. Pendlebury ST, Cuthbertson FC, Welch SJ, Mehta Z, Rothwell PM. Underestimation of cognitive impairment by mini-mental state examination versus the Montreal cognitive assessment in patients with transient ischemic attack and stroke a population-based study. Stroke. 2010;41(6):1290-1293.

28. Shega JW, Sunkara PD, Kotwal A, et al. Measuring Cognition: The Chicago Cognitive Function Measure in the National Social Life, Health and Aging Project, Wave 2. J Gerontol Ser B Psychol Sci Soc Sci. 2014;69(S2):S166-S176.

29. Cornwell B. Good health and the bridging of structural holes. Soc Networks. 2009;31(1):92-103.

30. Fuhrer R, Stansfeld SA. How gender affects patterns of social relations and their impact on health: a comparison of one or multiple sources of support from "close persons". Soc Sci Med. 2002;54(5):811-825.

31. Cornwell B. Independence through social networks: bridging potential among older women and men. J Gerontol Ser B Psychol Sci Soc Sci. 2011;66(6):782-794.

32. Umberson D. Gender, marital status and the social control of health behavior. Soc Sci Med. 1992;34(8):907-917.

33. Waite L, Gallagher M. The case for marriage: Why married people are happier, healthier and better off financially: Broadway Books; 2002.

34. Zhang Z . Gender differentials in cognitive impairment and decline of the oldest old in China. J Gerontol Ser B Psychol Sci Soc Sci. 2006;61(2):S107S115.

35. Cornwell EY, Waite LJ. Measuring social isolation among older adults using multiple indicators from the NSHAP study. J Gerontol Ser B Psychol Sci Soc Sci. 2009;64(suppl 1):i38. 
36. Cornwell B, Schumm LP, Laumann EO, Graber J. Social networks in the NSHAP study: rationale, measurement, and preliminary findings. J Gerontol Ser B Psychol Sci Soc Sci. 2009;64(suppl 1):i47.

37. Cronbach LJ. Coefficient alpha and the internal structure of tests. Psychometrika. 1951;16(3):297-334.

38. Nasreddine ZS, Phillips NA, Bédirian V, et al. The Montreal cognitive assessment, MoCA: a brief screening tool for mild cognitive impairment. J Am Geriatr Soc. 2005;53(4):695-699.

39. Kotwal AA, Schumm LP, Kern D, et al. Evaluation of a brief survey instrument for assessing subtle differences in cognitive function among older adults. Alzheimer Dis Assoc Disord. 2015;29(4):317-324.

40. McLennan SN, Mathias JL, Brennan LC, Stewart S. Validity of the Montreal cognitive assessment (MoCA) as a screening test for mild cognitive impairment $(\mathrm{MCI})$ in a cardiovascular population. J Geriatr Psychiatry Neurol. 2011;24(1):33-38.

41. Damian AM, Jacobson SA, Hentz JG, et al. The Montreal cognitive assessment and the mini-mental state examination as screening instruments for cognitive impairment: item analyses and threshold scores. Dement Geriatr Cogn Disord. 2011;31(2):126-131.

42. Waldron-Perrine B, Axelrod BN. Determining an appropriate cutting score for indication of impairment on the Montreal cognitive assessment. Int $\mathbf{j}$ Geriatric Psychiatry. 2012;27(11):1189-1194.

43. Freitas S, Simões MR, Alves L, Santana I. Montreal cognitive assessment: validation study for mild cognitive impairment and Alzheimer disease. Alzheimer Dis Assoc Disord. 2013;27(1):37-43.

44. Luis CA, Keegan AP, Mullan M. Cross validation of the Montreal cognitive assessment in community dwelling older adults residing in the southeastern US. Int J Geriatric Psychiatry. 2009;24(2):197-201.

45. Barnes DE, Cauley JA, Lui LY, et al. Women who maintain optimal cognitive function into old age. J Am Geriatr Soc. 2007;55(2):259 264.
46. Vasilopoulos T, Kotwal A, Huisingh-Scheetz MJ, Waite LJ, McClintock MK, Dale W. Comorbidity and chronic conditions in the national social life, health and aging project (NSHAP), wave 2. J Gerontol Ser B Psychol Sci Soc Sci. 2014;69(Suppl 2):S154-S165.

47. Payne C, Hedberg E, Kozloski M, Dale W, McClintock MK. Using and interpreting mental health measures in the national social life, health, and aging project. J Gerontol Ser B Psychol Sci Soc Sci. 2014;69(Suppl 2):S99S116.

48. Coups EJ, Gaba A, Orleans CT. Physician screening for multiple behavioral health risk factors. Am J Prev Med. 2004;27(2 Suppl):34.

49. Weisberg S. Applied linear regression. Vol 528: Wiley; 2005.

50. O'Muircheartaigh C, Eckman S, Smith S. Statistical design and estimation for the national social life, health, and aging project. J Gerontol: Series B. 2009;64(Supplement 1):i12.

51. Stata Statistical Software: Release 12 [computer program]. College Station: StataCorp LP; 2011.

52. Coleman JS. Social capital in the creation of human capital. Am J Sociol. 1988;94:S95-S120.

53. Cornwell B, Laumann EO, Schumm LP. The social connectedness of older adults: a national profile. Am Sociol Rev. 2008;73(2): 185-203.

54. Covinsky KE, Newcomer R, Fox $\mathbf{P}$, et al. Patient and caregiver characteristics associated with depression in caregivers of patients with dementia. J Gen Intern Med. 2003;18(12):1006-1014.

55. Schulz R, Beach SR. Caregiving as a risk factor for mortality: the caregiver health effects study. JAMA. 1999;282(23):2215-2219.

56. Schulz R, Martire LM. Family caregiving of persons with dementia: prevalence, health effects, and support strategies. Am J Geriatr Psychiatry. 2004; 12(3):240-249.

57. Salthouse T. All data collection and analysis methods have limitations: reply to rabbitt (2011) and Raz and lindenberger (2011). Psychol Bull. 2011;137(5):796-799. 